\title{
SOME FURTHER ARITHMETICAL IDENTITIES INVOLVING A GENERALIZATION OF RAMANUJAN'S SUM
}

\author{
Pentti Haukkanen
}

\section{Introduction}

Let $G$ be a commutative semigroup with identity $\mathbf{1}$, with respect to a multiplication denoted by juxtaposition. Suppose there exists a finite or countable infinite set $P(\subseteq G)$ of primes such that each $\mathbf{n} \in G$ can be represented uniquely in the form

$$
\mathbf{n}=\prod_{\mathbf{p} \in P} \mathbf{p}^{\mathbf{n}(\mathbf{p})}
$$

where the exponents $\mathbf{n}(\mathbf{p})$ are non-negative integers of which all but a finite number are zero. (Define $\mathbf{1}(\mathbf{p})=0$ for all $\mathbf{p} \in P$.) Further, suppose there exists a real-valued norm $\|\cdot\|$ defined on $G$ such that

(i) $\|\mathbf{1}\|=1, \quad\|\mathbf{p}\|>1 \quad(\mathbf{p} \in P)$,

(ii) $\|\mathbf{m} \mathbf{n}\|=\|\mathbf{m}\|\|\mathbf{n}\| \quad(\mathbf{m}, \mathbf{n} \in G)$,

(iii) the set $\{\mathbf{n} \in G:\|\mathbf{n}\| \leq x\}$ is finite for all real numbers $x$.

Then $G$ is called $[18$, p. 11] an arithmetical semigroup. Throughout this paper elements in an arbitrary arithmetical semigroup are typed in boldface.

Let $G$ be an arbitrary but fixed arithmetical semigroup. By an arithmetical function we mean a complex-valued function defined on the arithmetical semigroup $G$. Let $A$ be a mapping from the set $G$ into the set of subsets of $G$ such that for each $\mathbf{n} \in G, A(\mathbf{n})$ is a subset of the set of divisors of $\mathbf{n}$. Then the $A$-convolution of two arithmetical functions $f$ and $g$ is defined by

$$
(f A g)=\sum_{\mathbf{d} \in A(\mathbf{n})} f(\mathbf{d}) g(\mathbf{n} / \mathbf{d}) .
$$

In this paper we confine ourselves to regular convolutions and we shall assume the reader to be familiar with this notion (see e.g. [13, Section 1.3], [22, Chapter 4], [27]). For example, the Dirichlet convolution $D$, where $D(\mathbf{n})$ is the set of all divisors of $\mathbf{n}$, and the unitary convolution $U$, where $U(\mathbf{n})=\{\mathbf{d}: \mathbf{d} \mid \mathbf{n},(\mathbf{d}, \mathbf{n} / \mathbf{d})$ $=1\}$, are regular. 
For a positive integer $k$, we define

$$
A_{k}(\mathbf{n})=\left\{\mathbf{d} \in G: \mathbf{d}^{k} \in A\left(\mathbf{n}^{k}\right)\right\} .
$$

It has been shown (see [13, p. 10], [32, p. 267]) that the $A_{k}$-convolution is regular whenever the $A$-convolution is regular. The symbol $(\mathbf{a}, \mathbf{b})_{A, k}$ denotes the greatest $k$ th power divisor of a which belongs to $A(\mathbf{b})$.

The classical Ramanujan's sum $C(n ; r)$ is defined by

$$
C(n ; r)=\sum_{\substack{m(\bmod r) \\(m, r)=1}} \exp (2 \pi i m n / r)
$$

where $n$ is a non-negative integer and $r$ is a positive integer. Its well-known arithmetical representation is given by

$$
C(n ; r)=\sum_{d \mid(n, r)} d \mu(r / d)
$$

In [14] the author together with P.J. McCarthy defined a generalized Ramanujan's sum by

$$
C_{A, k}\left(n_{1}, \ldots, n_{u} ; r\right)=\sum_{\substack{m_{1}, \ldots, m_{u}\left(\bmod r^{k}\right) \\\left(\left(m_{i}\right), r^{k}\right)_{A, k}=1}} \exp \left(2 \pi i\left(m_{1} n_{1}+\cdots+m_{u} n_{u}\right) / r^{k}\right)
$$

where $n_{1}, \ldots, n_{u}$ are non-negative integers, $r$ is a positive integer and $\left(m_{i}\right)=$ $\left(m_{1}, \ldots, m_{\boldsymbol{u}}\right)$, the greatest common divisor of $m_{1}, \ldots, m_{\boldsymbol{u}}$. We noted [14] that

$$
C_{A, k}\left(n_{1}, \ldots, n_{u} ; r\right)=\sum_{d^{k} \in A\left(\left(\left(n_{i}\right), r^{k}\right)_{A, k}\right)} d^{k u} \mu_{A_{k}}(r / d)=\sum_{\substack{d \in A_{k}(r) \\ d^{k} \mid\left(n_{i}\right)}} d^{k u} \mu_{A_{k}}(r / d),
$$

where $\mu_{A_{k}}$ is the inverse of $E$, the function $\equiv 1$, with respect to the $A_{k}$ convolution. For an arithmetical semigroup this suggests we define

$$
C_{A, k}\left(\mathbf{n}_{1}, \ldots, \mathbf{n}_{u} ; \mathbf{r}\right)=\sum_{\mathbf{d}^{k} \in A\left(\left(\left(\mathbf{n}_{i}\right), \mathbf{r}^{k}\right)_{A, k}\right)}\|\mathrm{d}\|^{k u} \mu_{A_{k}}(\mathbf{r} / \mathbf{d})
$$

In [13] we defined a generalized Ramanujan's sum by

$$
S_{A, k}^{f, g}\left(\mathbf{n}_{1}, \ldots, \mathbf{n}_{u} ; \mathbf{r}\right)=\sum_{\mathbf{d}^{k} \in A\left(\left(\left(\mathbf{n}_{i}\right), \mathbf{r}^{k}\right)_{A, k}\right)} f(\mathbf{d}) g(\mathbf{r} / \mathbf{d})
$$


In other words,

$$
S_{A, k}^{f, g}\left(\mathbf{n}_{1}, \ldots, \mathbf{n}_{u} ; \mathbf{r}\right)=\sum_{\substack{\mathbf{d} \in A_{k}(\mathbf{r}) \\ \mathbf{d}^{k} \mid\left(\mathbf{n}_{i}\right)}} f(\mathbf{d}) g(\mathbf{r} / \mathbf{d})=\left(\chi\left(\left(\mathbf{n}_{i}\right) ;(\cdot)^{k}\right) f A_{k} g\right)(\mathbf{r}),
$$

where $\chi(\mathbf{n} ; \mathbf{d})=1$ if $\mathbf{d} \mid \mathbf{n}$, and $=0$ otherwise.

The purpose of [13] was to derive arithmetical identities of classical type involving that sum. The purpose of the present paper is to give more arithmetical identities for that sum. We shall also list a large number of known special cases of the given identities. At the end of this paper we shall note that two identities here can be extended to totally $A$ - $k$-even functions $(\bmod \mathbf{r})$.

\section{Preliminaries}

We define an arithmetical function $f$ to be quasi- $A$-multiplicative [13, p. 14] if $f(\mathbf{1}) \neq 0$ and $f(\mathbf{1}) f(\mathbf{m} \mathbf{n})=f(\mathbf{m}) f(\mathbf{n})$ whenever $\mathbf{m}, \mathbf{n} \in A(\mathbf{m n})$. Quasi- $A$ multiplicative functions $f$ with $f(\mathbf{1})=1$ are called $A$-multiplicative [45]. It is easy to see that an arithmetical function $f$ with $f(\mathbf{1}) \neq 0$ is quasi- $A$-multiplicative if, and only if, $f / f(\mathbf{1})$ is $A$-multiplicative. Quasi- $U$-multiplicative functions are called quasi-multiplicative [19]. For those functions $f(\mathbf{1}) \neq 0$ and $f(\mathbf{1}) f(\mathbf{m n})=$ $f(\mathbf{m}) f(\mathbf{n})$ whenever $(\mathbf{m}, \mathbf{n})=\mathbf{1}$. All quasi- $A$-multiplicative functions are quasimultiplicative.

Let $A^{(1)}, A^{(2)}, \ldots, A^{(u)}$ be regular convolutions. Then we define the $A^{(1)}$ $A^{(2)} \cdots A^{(u)}$-convolution of arithmetical functions $f\left(\mathbf{n}_{1}, \mathbf{n}_{2}, \ldots, \mathbf{n}_{u}\right)$ and $g\left(\mathbf{n}_{1}, \mathbf{n}_{2}\right.$, $\left.\ldots, \mathbf{n}_{u}\right)$ by

$$
\begin{aligned}
f\left(\mathbf{n}_{1}, \mathbf{n}_{2}, \ldots, \mathbf{n}_{u}\right) A^{(1)} A^{(2)} \cdots A^{(u)} g\left(\mathbf{n}_{1}, \mathbf{n}_{2}, \ldots, \mathbf{n}_{u}\right) \\
=\sum_{\mathbf{d}_{1} \in A^{(1)}\left(\mathbf{n}_{1}\right) \mathbf{d}_{2} \in A^{(2)}\left(\mathbf{n}_{2}\right)} \sum_{\mathbf{d}_{u} \in A^{(u)}\left(\mathbf{n}_{u}\right)} f\left(\mathbf{d}_{1}, \mathbf{d}_{2}, \ldots, \mathbf{d}_{u}\right) . \\
\cdot g\left(\mathbf{n}_{1} / \mathbf{d}_{1}, \mathbf{n}_{2} / \mathbf{d}_{2}, \ldots, \mathbf{n}_{u} / \mathbf{d}_{u}\right) .
\end{aligned}
$$

It is easy to see that an $A^{(1)} A^{(2)} \cdots A^{(u)}$-convolution of arithmetical functions is associative. Also, if $h$ is an $A^{(i)}$-multiplicative function, then

$$
\begin{aligned}
\left(f\left(\mathbf{n}_{1}, \mathbf{n}_{2}, \ldots, \mathbf{n}_{u}\right) A^{(1)} A^{(2)} \ldots A^{(u)} g\left(\mathbf{n}_{1}, \mathbf{n}_{2}, \ldots, \mathbf{n}_{u}\right)\right) h\left(\mathbf{n}_{i}\right) \\
\quad=f\left(\mathbf{n}_{1}, \mathbf{n}_{2}, \ldots, \mathbf{n}_{u}\right) h\left(\mathbf{n}_{i}\right) A^{(1)} A^{(2)} \ldots A^{(u)} g\left(\mathbf{n}_{1}, \mathbf{n}_{2}, \ldots, \mathbf{n}_{u}\right) h\left(\mathbf{n}_{i}\right) .
\end{aligned}
$$

Let $f$ be an arithmetical function of one variable and $e, m, u \in \mathbf{N}, u \geq 2$, $0 \leq m<u$. Then we define $P_{e}(f)\left(\mathbf{n}_{1}, \ldots, \mathbf{n}_{m} ; \mathbf{n}_{m+1}, \ldots, \mathbf{n}_{u}\right)$ to be the arithmetical function of $u$ variables such that

$$
P_{e}(f)\left(\mathbf{n}_{1}, \ldots, \mathbf{n}_{m} ; \mathbf{n}_{m+1}, \ldots, \mathbf{n}_{u}\right)=\left\{\begin{array}{c}
f\left(\mathbf{n}_{u}\right), \\
\begin{array}{c}
\text { if } \mathbf{n}_{1}=\cdots=\mathbf{n}_{m}=\left(\mathbf{n}_{m+1}\right)^{e} \\
=\cdots=\left(\mathbf{n}_{u}\right)^{e}
\end{array} \\
\text { otherwise }
\end{array}\right.
$$


In particular, we denote

$$
P_{1}(f)\left(\mathbf{n}_{1}, \ldots, \mathbf{n}_{m} ; \mathbf{n}_{m+1}, \ldots, \mathbf{n}_{u}\right)=P(f)\left(\mathbf{n}_{1}, \ldots, \mathbf{n}_{u}\right) .
$$

If $m=0$, then we have

$$
P_{e}(f)\left(\mathbf{n}_{1}, \ldots, \mathbf{n}_{m} ; \mathbf{n}_{m+1}, \ldots, \mathbf{n}_{u}\right)=P(f)\left(\mathbf{n}_{1}, \ldots, \mathbf{n}_{u}\right) .
$$

We note that some special cases of the function $P_{e}(f)$ can be found in $[39$, p. 627] and $[42$, p. 86].

It is easy to see that if $f, g$ are arithmetical functions of one variable and $u \geq 2,1 \leq i \leq u$, then

$$
g\left(\mathbf{n}_{i}\right) P(f)\left(\mathbf{n}_{1}, \mathbf{n}_{2}, \ldots, \mathbf{n}_{u}\right)=P(f g)\left(\mathbf{n}_{1}, \mathbf{n}_{2}, \ldots, \mathbf{n}_{u}\right) .
$$

Also, if $f, g$ are arithmetical functions of one variable, $1 \leq j \leq u$ and

$$
A^{(j)}(\mathbf{n}) \subseteq A^{(i)}(\mathbf{n})
$$

whenever $\mathbf{n} \in G, 1 \leq i \leq u, i \neq j$, then

$$
\begin{gathered}
P(f)\left(\mathbf{n}_{1}, \mathbf{n}_{2}, \ldots, \mathbf{n}_{u}\right) A^{(1)} A^{(2)} \ldots A^{(u)} P(g)\left(\mathbf{n}_{1}, \mathbf{n}_{2}, \ldots, \mathbf{n}_{u}\right) \\
=P\left(f A^{(j)} g\right)\left(\mathbf{n}_{1}, \mathbf{n}_{2}, \ldots, \mathbf{n}_{u}\right) .
\end{gathered}
$$

\section{Identities}

Theorem 1. Suppose $f$ and $g$ are arithmetical functions and $0 \leq m \leq u$. Then for $\mathbf{n}_{1}, \ldots, \mathbf{n}_{u}, \mathbf{r} \in G$

$$
\begin{aligned}
& S_{A, k}^{f, g}\left(\mathbf{n}_{1}, \ldots, \mathbf{n}_{m},\left(\mathbf{n}_{m+1}\right)^{k}, \ldots,\left(\mathbf{n}_{u}\right)^{k} ; \mathbf{r}\right) \\
& \quad=E\left(\mathbf{n}_{1}\right) \cdots E\left(\mathbf{n}_{u}\right) g(\mathbf{r}) D \cdots D A_{k} P_{k}(f)\left(\mathbf{n}_{1}, \ldots, \mathbf{n}_{m} ; \mathbf{n}_{m+1}, \ldots, \mathbf{n}_{u}, \mathbf{r}\right),
\end{aligned}
$$

where $E(\mathbf{n})=1$ for all $\mathbf{n} \in G$.

Proof. By (1) and (3),

$$
\begin{aligned}
& E\left(\mathbf{n}_{1}\right) \cdots E\left(\mathbf{n}_{u}\right) g(\mathbf{r}) D \cdots D A_{k} P_{k}(f)\left(\mathbf{n}_{1}, \ldots, \mathbf{n}_{m} ; \mathbf{n}_{m+1}, \ldots, \mathbf{n}_{u}, \mathbf{r}\right) \\
& =\sum_{\mathbf{d}_{1} \mid \mathbf{n}_{1}} \cdots \sum_{\mathbf{d}_{u} \mid \mathbf{n}_{u}} \sum_{\mathbf{d} \in A_{k}(\mathbf{r})} E\left(\mathbf{n}_{1} / \mathbf{d}_{1}\right) \cdots E\left(\mathbf{n}_{u} / \mathbf{d}_{u}\right) g(\mathbf{r} / \mathbf{d}) \\
& \text { - } P_{k}(f)\left(\mathbf{d}_{1}, \ldots, \mathbf{d}_{m} ; \mathbf{d}_{m+1}, \ldots, \mathbf{d}_{u}, \mathbf{d}\right) \\
& =\sum_{\substack{\mathbf{d} \in A_{k}(\mathbf{r}), \mathbf{d}\left|\mathbf{n}_{m+1}, \ldots, \mathbf{n}_{u} \\
\mathbf{d}^{k}\right| \mathbf{n}_{1}, \ldots, \mathbf{n}_{m}}} g(\mathbf{r} / \mathbf{d}) P_{k}(f)\left(\mathbf{d}^{k}, \ldots, \mathbf{d}^{k} ; \mathbf{d}, \ldots, \mathbf{d}, \mathbf{d}\right) \\
& =\sum_{\substack{\mathbf{d} \in A_{k}(\mathbf{r}), \mathbf{d}\left|\mathbf{n}_{m+1}, \ldots, \mathbf{n}_{u} \\
\mathbf{d}^{k}\right| \mathbf{n}_{1}, \ldots, \mathbf{n}_{m}}} g(\mathbf{r} / \mathbf{d}) f(\mathbf{d}) \\
& =S_{A, k}^{f, g}\left(\mathbf{n}_{1}, \ldots, \mathbf{n}_{m},\left(\mathbf{n}_{m+1}\right)^{k}, \ldots,\left(\mathbf{n}_{u}\right)^{k} ; \mathbf{r}\right),
\end{aligned}
$$

which was to be proved. 
Theorem 2. Suppose $h_{1}, \ldots, h_{u}$ are quasi- $D$-multiplicative functions, $h$ is a quasi- $A_{k}$-multiplicative function and $f, g, H$ are arbitrary arithmetical functions. Then for $\mathbf{n}_{1}, \mathbf{n}_{2}, \ldots, \mathbf{n}_{u}, \mathbf{r} \in G$

$$
\begin{aligned}
\left(h_{1} \cdots h_{u} h\right)(\mathbf{1}) \sum_{\substack{\mathbf{d} \in A_{k}(\mathbf{r}) \\
\mathbf{d} \mid\left(\mathbf{n}_{i}\right)}} S_{A, k}^{f, g}\left(\left(\mathbf{n}_{1} / \mathbf{d}\right)^{k}, \ldots,\left(\mathbf{n}_{u} / \mathbf{d}\right)^{k} ; \mathbf{r} / \mathbf{d}\right) \\
\quad \cdot h_{1}\left(\mathbf{n}_{1} / \mathbf{d}\right) \cdots h_{u}\left(\mathbf{n}_{u} / \mathbf{d}\right) h(\mathbf{r} / \mathbf{d}) H(\mathbf{d}) \\
=\sum_{\substack{\mathbf{d} \in A_{k}(\mathbf{r}) \\
\mathbf{d} \mid\left(\mathbf{n}_{i}\right)}} h_{1}\left(\mathbf{n}_{1} / \mathbf{d}\right) \cdots h_{u}\left(\mathbf{n}_{u} / \mathbf{d}\right)(h g)(\mathbf{r} / \mathbf{d})\left(\left(f h_{1} \cdots h_{u} h\right) A_{k} H\right)(\mathbf{d}) .
\end{aligned}
$$

Proof. It suffices to consider the case $h_{1}(\mathbf{1})=\cdots=h_{u}(\mathbf{1})=h(\mathbf{1})=1$. Let $L$ denote the left-hand side of the identity in Theorem 2 . Then, by (1)-(6) and Theorem 1,

$$
\begin{aligned}
L= & \left(E\left(\mathbf{n}_{1}\right) \cdots E\left(\mathbf{n}_{u}\right) g(\mathbf{r})_{D} \cdots D A_{k} P(f)\left(\mathbf{n}_{1}, \mathbf{n}_{2}, \ldots, \mathbf{n}_{u}, \mathbf{r}\right)\right) \\
& \cdot h_{1}\left(\mathbf{n}_{1}\right) \cdots h_{u}\left(\mathbf{n}_{u}\right) h(\mathbf{r})_{D} \cdots D A_{k} P(H)\left(\mathbf{n}_{1}, \mathbf{n}_{2}, \ldots, \mathbf{n}_{u}, \mathbf{r}\right) \\
= & \left(h_{1}\left(\mathbf{n}_{1}\right) \cdots h_{u}\left(\mathbf{n}_{u}\right)(h g)(\mathbf{r})_{D} \cdots D A_{k} h_{1}\left(\mathbf{n}_{1}\right) \cdots h_{u}\left(\mathbf{n}_{u}\right) h(\mathbf{r})\right. \\
& \left.\cdot P(f)\left(\mathbf{n}_{1}, \mathbf{n}_{2}, \ldots, \mathbf{n}_{u}, \mathbf{r}\right)\right) D \cdots D A_{k} P(H)\left(\mathbf{n}_{1}, \mathbf{n}_{2}, \ldots, \mathbf{n}_{u}, \mathbf{r}\right) \\
= & \left(h_{1}\left(\mathbf{n}_{1}\right) \cdots h_{u}\left(\mathbf{n}_{u}\right)(h g)(\mathbf{r})_{D} \cdots D A_{k} P\left(f h_{1} \cdots h_{u} h\right)\left(\mathbf{n}_{1}, \mathbf{n}_{2}, \ldots, \mathbf{n}_{u}, \mathbf{r}\right)\right) \\
& \cdot D \cdots D A_{k} P(H)\left(\mathbf{n}_{1}, \mathbf{n}_{2}, \ldots, \mathbf{n}_{u}, \mathbf{r}\right) \\
= & h_{1}\left(\mathbf{n}_{1}\right) \cdots h_{u}\left(\mathbf{n}_{u}\right)(h g)(\mathbf{r})_{D} \cdots D A_{k} \\
& \cdot\left(P\left(f h_{1} \cdots h_{u} h\right)\left(\mathbf{n}_{1}, \mathbf{n}_{2}, \ldots, \mathbf{n}_{u}, \mathbf{r}\right)_{D} \cdots D A_{k} P(H)\left(\mathbf{n}_{1}, \mathbf{n}_{2}, \ldots, \mathbf{n}_{u}, \mathbf{r}\right)\right) \\
= & h_{1}\left(\mathbf{n}_{1}\right) \cdots h_{u}\left(\mathbf{n}_{u}\right)(h g)(\mathbf{r})_{D} \cdots D A_{k} P\left(\left(f h_{1} \cdots h_{u} h\right)_{A_{k}} H\right)\left(\mathbf{n}_{1}, \mathbf{n}_{2}, \ldots, \mathbf{n}_{u}, \mathbf{r}\right) .
\end{aligned}
$$

We thus arrive at our result.

Theorem 3. Suppose $H$ is a quasi- $A_{k}$-multiplicative function, $H_{1}, H_{2}$, $\ldots, H_{u}$ are quasi- $D$-multiplicative functions and $f, g, h, h_{1}, h_{2}, \ldots, h_{u}$ are arbitrary arithmetical functions. Then for $\mathbf{n}_{1}, \mathbf{n}_{2}, \ldots, \mathbf{n}_{u}, \mathbf{r} \in G, a_{1}, \ldots, a_{u}=0,1$ 


$$
\begin{aligned}
\left(H_{1} \cdots\right. & \left.H_{u} H\right)(\mathbf{1}) \sum_{\mathbf{d}_{1} \mid \mathbf{n}_{1}} \cdots \sum_{\mathbf{d}_{u} \mid \mathbf{n}_{u}} \sum_{\delta \in A_{k}(\mathbf{r})} S_{A, k}^{f, g}\left(\mathbf{d}_{1}^{k^{a_{1}}}, \ldots, \mathbf{d}_{u}^{k^{a_{u}}} ; \boldsymbol{\delta}\right) \\
& \cdot H_{1}\left(\mathbf{d}_{1}\right) \cdots H_{u}\left(\mathbf{d}_{u}\right) H(\delta) h_{1}\left(\mathbf{n}_{1} / \mathbf{d}_{1}\right) \cdots h_{u}\left(\mathbf{n}_{u} / \mathbf{d}_{u}\right) h(\mathbf{r} / \delta) \\
= & \sum_{\substack{\mathbf{d} \in A_{k}(\mathbf{r}) \\
\mathbf{d}^{k} \mid\left(\mathbf{n}_{i}^{k^{a_{i}}}\right)}}(f H)(\mathbf{d}) H_{1}\left(\mathbf{d}^{k^{1-a_{1}}}\right) \cdots H_{u}\left(\mathbf{d}^{k^{1-a_{u}}}\right)\left(h A_{k}(g H)\right)(\mathbf{r} / \mathbf{d}) \\
& \cdot\left(H_{1} D h_{1}\right)\left(\mathbf{n}_{1} / \mathbf{d}^{k^{1-a_{1}}}\right) \cdots\left(H_{u} D h_{u}\right)\left(\mathbf{n}_{u} / \mathbf{d}^{k^{1-a_{u}}}\right) .
\end{aligned}
$$

Proof. It suffices to consider the case $H_{1}(\mathbf{1})=\cdots=H_{u}(\mathbf{1})=H(\mathbf{1})=1$. Further, without loss of generality we may assume $a_{1}=\ldots=a_{m}=0, a_{m+1}=$ $\cdots=a_{u}=1(0 \leq m \leq u)$. Then, by (1), the left-hand side of the identity in Theorem 3 can be written as

$$
\begin{aligned}
h_{1}\left(\mathbf{n}_{1}\right) & \cdots h_{u}\left(\mathbf{n}_{u}\right) h(\mathbf{r}) D \ldots D A_{k} S_{A, k}^{f, g}\left(\mathbf{n}_{1}, \ldots, \mathbf{n}_{m},\left(\mathbf{n}_{m+1}\right)^{k}, \ldots,\left(\mathbf{n}_{u}\right)^{k} ; \mathbf{r}\right) \\
& \cdot H_{1}\left(\mathbf{n}_{1}\right) \cdots H_{u}\left(\mathbf{n}_{u}\right) H(\mathbf{r}) .
\end{aligned}
$$

Thus, applying Theorem 1 and formulas (2), (3), we have the theorem.

Remark. The functions $h_{1}, \ldots, h_{u}, h$ are of great importance in Theorem 3. In fact, if $h=E_{0}$, defined by $E_{0}(\mathbf{1})=1$ and $E_{0}(\mathbf{n})=0$ for $\mathbf{n} \neq \mathbf{1}$, then the summation over $\mathbf{d}_{1}, \ldots, \mathbf{d}_{u}, \delta$ reduces to the summation over $\mathbf{d}_{1}, \ldots, \mathbf{d}_{u} . \mathrm{A}$ similar reduction is valid with respect to any subset of the set of functions $h_{1}, \ldots$, $h_{u}, h$.

Notation. For an arithmetical function $f$, denote

$$
\begin{gathered}
f^{\wedge}(A, \mathbf{e} ; x)=\sum_{\substack{\|\mathbf{n}\| \leq x \\
\mathbf{n} \in A(\mathbf{n} \mathbf{e})}} f(\mathbf{n}) \quad(x \in \mathbf{R}), \\
f^{\wedge}(x)=f^{\wedge}(D, \mathbf{e} ; x)=\sum_{\|\mathbf{n}\| \leq x} f(\mathbf{n}) \quad(x \in \mathbf{R}) .
\end{gathered}
$$

Theorem 4. Suppose $f, g, h, h_{1}, h_{2}, \ldots, h_{m}(0 \leq m \leq u)$ are arithmetical functions. Then for $x_{1}, \ldots, x_{m}, y>0\left(x_{1}, \ldots, x_{m}, y \in \mathbf{R}\right), \mathbf{n}_{m+1}, \ldots, \mathbf{n}_{u}$, $\mathbf{r} \in G, a_{1}, \ldots, a_{m}=0,1$

$$
\sum_{\left\|\mathbf{i}_{1}\right\| \leq x_{1}} \cdots \sum_{\left\|\mathbf{i}_{m}\right\| \leq x_{m}} \sum_{\|\mathbf{j}\| \leq y} S_{A, k}^{f, g}\left(\mathbf{i}_{1}^{k^{a_{1}}}, \ldots, \mathbf{i}_{m}^{k^{a_{m}}}, \mathbf{n}_{m+1}, \ldots, \mathbf{n}_{u} ; \mathbf{j}\right)
$$


Arithmetical identities involving a generalization of Ramanujan's sum

$$
\begin{aligned}
& \cdot h_{1}^{\wedge}\left(x_{1} /\left\|\mathbf{i}_{1}\right\|\right) \cdots h_{m}^{\wedge}\left(x_{m} /\left\|\mathbf{i}_{m}\right\|\right) h^{\wedge}\left(A_{k}, \mathbf{j} ; y /\|\mathbf{j}\|\right)
\end{aligned}
$$

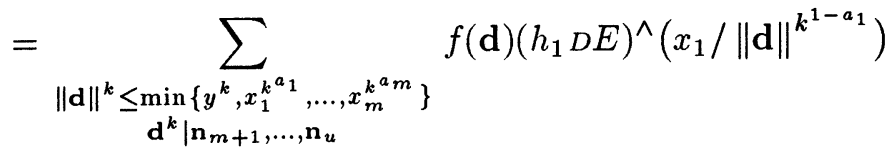

$$
\begin{aligned}
& \cdots\left(h_{m} D E\right)^{\wedge}\left(x_{m} /\|\mathbf{d}\|^{k^{1-a_{m}}}\right)\left(h_{A_{k}} g\right)^{\wedge}\left(A_{k}, \mathbf{d} ; y /\|\mathbf{d}\|\right) \text {, }
\end{aligned}
$$

(8)

$$
\begin{aligned}
\sum_{\left\|\mathbf{i}_{1}\right\| \leq x_{1}} \ldots \sum_{\substack{\left\|\mathbf{i}_{m}\right\| \leq x_{m} \\
. h_{1}^{\wedge}\left(x_{1} /\left\|\mathbf{i}_{1}\right\|\right)}} S_{A, k}^{f, g}\left(\mathbf{i}_{1}^{k^{a_{1}}}, \ldots, h_{m}^{\wedge}\left(x_{m} /\left\|\mathbf{i}_{m}^{k_{m}}\right\|\right)\right. \\
\left.=\sum_{\substack{\|\mathbf{d}\|^{k} \leq \min \\
\mathbf{d}^{k} \mid \mathbf{n}_{m+1}, \ldots, \mathbf{n}_{u} ; \mathbf{d} \in A_{k}(\mathbf{r})}} f(\mathbf{d}) g(\mathbf{r} / \mathbf{d})\left(h_{1} D E\right)^{\wedge}\left(x_{1} /\|\mathbf{d}\|^{k^{1-a_{1}}}\right) \ldots, \mathbf{n}_{u} ; \mathbf{r}\right) \\
\\
\cdot\left(h_{m} D E\right)^{\wedge}\left(x_{m} /\|\mathbf{d}\|^{k^{a_{m} a_{m}}}\right), \quad m \geq 1 .
\end{aligned}
$$

Proof. Let $L$ denote the left-hand side of (7). Then, using the notation of $\chi$ given in the introduction, we can write

$$
\begin{aligned}
L= & \sum_{\left\|\mathbf{i}_{1}\right\| \leq x_{1}} \cdots \sum_{\left\|\mathbf{i}_{m}\right\| \leq x_{m}} \sum_{\|\mathbf{j}\| \leq y} \sum_{\substack{\mathbf{d} \in A_{k}(\mathbf{j}) \\
\mathbf{d}^{k^{1-a_{\nu}} \mid \mathbf{i}_{\nu}, \nu=1, \ldots, m}}} \chi\left(\left(\mathbf{n}_{m+1}, \ldots, \mathbf{n}_{u}\right) ; \mathbf{d}^{k}\right) \\
& \cdot f(\mathbf{d}) g(\mathbf{j} / \mathbf{d}) \sum_{\left\|\mathbf{b}_{1}\right\| \leq x_{1} /\left\|\mathbf{i}_{1}\right\|} h_{1}\left(\mathbf{b}_{1}\right) \cdots \sum_{\left\|\mathbf{b}_{m}\right\| \leq x_{m} /\left\|\mathbf{i}_{m}\right\|} h_{m}\left(\mathbf{b}_{m}\right) \sum_{\substack{\|\mathbf{a}\| \leq y /\|\mathbf{j}\| \\
a \in A_{k}(\mathbf{j a})}} h(\mathbf{a}) .
\end{aligned}
$$

Now we shall change the order of summation. It can be proved that the rule

$$
\left(\mathbf{c}_{1}, \ldots, \mathbf{c}_{m}, \mathbf{v}, \mathbf{d}, \mathbf{b}_{1}, \ldots, \mathbf{b}_{m}, \mathbf{a}\right) \rightarrow\left(\mathbf{c}_{1} / \mathbf{b}_{1}, \ldots, \mathbf{c}_{m} / \mathbf{b}_{m}, \mathbf{v} / \mathbf{a}, \mathbf{d}, \mathbf{b}_{1}, \ldots, \mathbf{b}_{m}, \mathbf{a}\right)
$$

defines a bijection from the set of $(2 m+3)$-tuples $\left(\mathbf{c}_{1}, \ldots, \mathbf{c}_{m}, \mathbf{v}, \mathbf{d}, \mathbf{b}_{1}, \ldots, \mathbf{b}_{m}, \mathbf{a}\right)$ satisfying

$$
\begin{gathered}
\left\|\mathbf{c}_{1}\right\| \leq x_{1}, \ldots,\left\|\mathbf{c}_{m}\right\| \leq x_{m}, \quad\|\mathbf{v}\| \leq y, \\
\mathbf{d} \in A_{k}(\mathbf{v}), \quad \mathbf{d}^{k^{1-a_{1}}}\left|\mathbf{c}_{1}, \ldots, \mathbf{d}^{k^{1-a_{m}}}\right| \mathbf{c}_{m}, \\
\mathbf{b}_{1}\left|\mathbf{c}_{1} \mathbf{d}^{-k^{1-a_{1}}}, \ldots, \mathbf{b}_{m}\right| \mathbf{c}_{m} \mathbf{d}^{-k^{1-a_{m}}}, \quad \mathbf{a} \in A_{k}(\mathbf{v} / \mathbf{d})
\end{gathered}
$$

onto the set of $(2 m+3)$-tuples $\left(\mathbf{i}_{1}, \ldots, \mathbf{i}_{m}, \mathbf{j}, \mathbf{d}, \mathbf{b}_{1}, \ldots, \mathbf{b}_{m}, \mathbf{a}\right)$ satisfying

$$
\left\|\mathbf{i}_{1}\right\| \leq x_{1}, \ldots,\left\|\mathbf{i}_{m}\right\| \leq x_{m}, \quad\|\mathbf{j}\| \leq y
$$




$$
\mathbf{d} \in A_{k}(\mathbf{j}), \quad \mathbf{d}^{k^{1-a_{1}}}\left|\mathbf{i}_{1}, \ldots, \mathbf{d}^{k^{1-a_{m}}}\right| \mathbf{i}_{m},
$$

$\left\|\mathbf{b}_{1}\right\| \leq x_{1} /\left\|\mathbf{i}_{1}\right\|, \ldots,\left\|\mathbf{b}_{m}\right\| \leq x_{m} /\left\|\mathbf{i}_{m}\right\|, \quad\|\mathbf{a}\| \leq y /\|\mathbf{j}\|, \quad \mathbf{a} \in A_{k}(\mathbf{j a})$.

Thus we obtain

$$
\begin{aligned}
L= & \sum_{\left\|\mathbf{c}_{1}\right\| \leq x_{1}} \ldots \sum_{\left\|\mathbf{c}_{m}\right\| \leq x_{m}} \sum_{\|\mathbf{v}\| \leq y} \sum_{\substack{\mathbf{d} \in A_{k}(\mathbf{v}) \\
\mathbf{d}^{k^{1-a}} \mid \mathbf{c}_{v}, v=1, \ldots, m}} \chi\left(\left(\mathbf{n}_{m+1}, \ldots, \mathbf{n}_{u}\right) ; \mathbf{d}^{k}\right) f(\mathbf{d}) \\
& \cdot \sum_{\mathbf{b}_{1} \mid \mathbf{c}_{\mathbf{1}} \mathbf{d}^{-k^{1-a_{1}}}} h_{1}\left(\mathbf{b}_{1}\right) \ldots \sum_{\left\|\mathbf{c}_{1}\right\| \leq x_{1}} \sum_{\| \mathbf{b}_{m} \mid \mathbf{c}_{m} \mathbf{d}^{-k^{1-a_{m}}}} h_{m}\left(\mathbf{b}_{m}\right) \sum_{\mathbf{a} \in A_{k}(\mathbf{v} / \mathbf{d})} h(\mathbf{a}) g((\mathbf{v} / \mathbf{d}) / \mathbf{a}) \\
= & \sum_{\substack{\mathbf{c}_{m} \\
\|\mathbf{v}\| \leq y}} \sum_{\substack{\mathbf{d} \in A_{k}(\mathbf{v}) \\
\mathbf{d}^{k^{1-a}} \mid \mathbf{c}_{v}, v=1, \ldots, m}} \chi\left(\left(\mathbf{n}_{m+1}, \ldots, \mathbf{n}_{u}\right) ; \mathbf{d}^{k}\right) f(\mathbf{d}) \\
& \cdot\left(h_{1} D E\right)\left(\mathbf{c}_{1} \mathbf{d}^{-k^{1-a_{1}}}\right) \cdots\left(h_{m} D E\right)\left(\mathbf{c}_{m} \mathbf{d}^{-k^{1-a_{m}}}\right)\left(h_{A_{k}} g\right)(\mathbf{v} / \mathbf{d}) .
\end{aligned}
$$

Further, it can be proved that the rule

$$
\left(\mathbf{e}_{1}, \ldots, \mathbf{e}_{m}, \mathbf{t}, \mathbf{d}\right) \rightarrow\left(\mathbf{e}_{1} \mathbf{d}^{k^{1-a_{1}}}, \ldots, \mathbf{e}_{m} \mathbf{d}^{k^{1-a_{m}}}, \mathbf{t d}, \mathbf{d}\right)
$$

defines a bijection from the set of $(m+2)$-tuples $\left(\mathbf{e}_{1}, \ldots, \mathbf{e}_{\boldsymbol{m}}, \mathbf{t}, \mathbf{d}\right)$ satisfying

$$
\|\mathbf{d}\|^{k} \leq \min \left\{y^{k}, x_{1}^{k^{a_{1}}}, \ldots, x_{m}^{k^{a_{m}}}\right\}
$$

$\left\|\mathbf{e}_{1}\right\| \leq x_{1} /\|\mathbf{d}\|^{k^{1-a_{1}}}, \ldots,\left\|\mathbf{e}_{m}\right\| \leq x_{m} /\|\mathbf{d}\|^{k^{1-a_{m}}}, \quad\|\mathbf{t}\| \leq y /\|\mathbf{d}\|, \quad \mathbf{t} \in A_{k}(\mathbf{t d})$, onto the set of $(m+2)$-tuples $\left(\mathbf{c}_{1}, \ldots, \mathbf{c}_{m}, \mathbf{v}, \mathbf{d}\right)$ satisfying $\left\|\mathbf{c}_{1}\right\| \leq x_{1}, \ldots,\left\|\mathbf{c}_{m}\right\| \leq x_{m}, \quad\|\mathbf{v}\| \leq y, \quad \mathbf{d} \in A_{k}(\mathbf{v}), \quad \mathbf{d}^{k^{1-a_{1}}}\left|\mathbf{c}_{1}, \ldots, \mathbf{d}^{k^{1-a_{m}}}\right| \mathbf{c}_{m}$. Thus

$$
\begin{aligned}
L= & \sum_{\|\mathbf{d}\|^{k} \leq \min \left\{y^{k}, x_{1}^{k^{a_{1}}}, \ldots, x_{m}^{k^{a_{m}}}\right\}} \chi\left(\left(\mathbf{n}_{m+1}, \ldots, \mathbf{n}_{u}\right) ; \mathbf{d}^{k}\right) f(\mathbf{d}) \\
& \cdot \sum_{\left\|\mathbf{e}_{1}\right\| \leq x_{1} /\|\mathbf{d}\|^{k^{1-a_{1}}}}\left(h_{1} D E\right)\left(\mathbf{e}_{1}\right) \cdots \sum_{\left\|\mathbf{e}_{m}\right\| \leq x_{m} /\|\mathbf{d}\|^{k^{1-a_{m}}}}\left(h_{m} D E\right)\left(\mathbf{e}_{m}\right) \\
& \cdot \sum_{\substack{\|\mathbf{t}\| \leq y /\|\mathbf{d}\| \\
\mathbf{t} \in A_{k}(\mathbf{t} \mathbf{d})}}\left(h_{\left.A_{k} g\right)(\mathbf{t})} \sum_{\substack{\|\mathbf{d}\|^{k} \leq \min \left\{y^{k}, x_{1}^{k^{a_{1}}}, \ldots, x_{m}^{k^{a_{m}}}\right\} \\
\mathbf{d}^{k} \mid \mathbf{n}_{m+1}, \ldots, \mathbf{n}_{u}}} f(\mathbf{d})\left(h_{1} D E\right)^{\wedge}\left(x_{1} /\|\mathbf{d}\|^{k^{1-a_{1}}}\right) \ldots\right. \\
& \cdot\left(h_{m} D E\right)^{\wedge}\left(x_{m} /\|\mathbf{d}\|^{k^{1-a_{m}}}\right)\left(h_{A_{k}} g\right)^{\wedge}\left(A_{k}, \mathbf{d} ; y /\|\mathbf{d}\|\right) .
\end{aligned}
$$

This proves (7). The proof of (8) goes through on similar lines. 
Theorem 5. Suppose $h_{1}, h_{2}, \ldots, h_{m}(0 \leq m \leq u)$ are quasi- $D$-multiplicative functions, $h$ is a quasi- $A_{k}$-multiplicative function and $f, g$ are arbitrary arithmetical functions. Then for $x_{1}, \ldots, x_{m}, y>0\left(x_{1}, \ldots, x_{m}, y \in \mathbf{R}\right), \mathbf{n}_{m+1}$, $\ldots, \mathbf{n}_{u}, \mathbf{r} \in G, a_{1}, \ldots, a_{m}=0,1$

$$
\begin{aligned}
(9)\left(h_{1} \cdots h_{m} h\right)(\mathbf{1}) \sum_{\left\|\mathbf{i}_{1}\right\| \leq x_{1}} \ldots \sum_{\left\|\mathbf{i}_{m}\right\| \leq x_{m}} \sum_{\|\mathbf{j}\| \leq y} S_{A, k}^{f, g}\left(\mathbf{i}_{1}^{k^{a_{1}}}, \ldots, \mathbf{i}_{m}^{k^{a_{m}}}, \mathbf{n}_{m+1}, \ldots, \mathbf{n}_{u} ; \mathbf{j}\right) \\
=h_{1}\left(\mathbf{i}_{1}\right) \cdots h_{m}\left(\mathbf{i}_{m}\right) h(\mathbf{j}) \\
\sum_{\substack{\|\mathbf{d}\|^{k} \leq \min \left\{\boldsymbol{y}^{k}, x_{1}^{k^{a_{1}}}, \ldots, \boldsymbol{x}_{m}^{k^{a_{m}}}\right\} \\
\mathbf{d}^{k} \mid \mathbf{n}_{m+1}, \ldots, \mathbf{n}_{u}}} f(\mathbf{d}) h(\mathbf{d}) h_{1}\left(\mathbf{d}^{k^{1-a_{1}}}\right) \cdots h_{m}\left(\mathbf{d}^{k^{1-a_{m}}}\right) \\
\cdot h_{1}^{\wedge}\left(x_{1} /\|\mathbf{d}\|^{k^{1-a_{1}}}\right) \cdots h_{m}^{\wedge}\left(x_{m} /\|\mathbf{d}\|^{k^{1-a_{m}}}\right)(g h)^{\wedge}\left(A_{k}, \mathbf{d} ; y /\|\mathbf{d}\|\right)
\end{aligned}
$$

$$
\begin{aligned}
& \left(h_{1} \cdots h_{m}\right)(\mathbf{1}) \sum_{\left\|\mathbf{i}_{1}\right\| \leq x_{1}} \ldots \sum_{\left\|\mathbf{i}_{m}\right\| \leq x_{m}} S_{A, k}^{f, g}\left(\mathbf{i}_{1}^{k^{a_{1}}}, \ldots, \mathbf{i}_{m}^{k^{a_{m}}}, \mathbf{n}_{m+1}, \ldots, \mathbf{n}_{u} ; \mathbf{r}\right) \\
& \cdot h_{1}\left(\mathbf{i}_{1}\right) \cdots h_{m}\left(\mathbf{i}_{m}\right) \\
& =\sum_{\substack{\|\mathbf{d}\|^{k} \leq \min \left\{\mathbf{x}_{1}^{k^{a_{1}}}, \ldots, \boldsymbol{x}_{m}^{k^{a_{m}}}\right\} \\
\mathbf{d}^{k} \mid \mathbf{n}_{m+1}, \ldots, \mathbf{n}_{u} ; \mathbf{d} \in A_{k}(\mathbf{r})}} f(\mathbf{d}) g(\mathbf{r} / \mathbf{d}) h_{1}\left(\mathbf{d}^{k^{1-a_{1}}}\right) \cdots h_{m}\left(\mathbf{d}^{k^{1-a_{m}}}\right) \\
& \cdot h_{1}^{\wedge}\left(x_{1} /\|\mathbf{d}\|^{k^{1-a_{1}}}\right) \cdots h_{m}^{\wedge}\left(x_{m} /\|\mathbf{d}\|^{k^{1-a_{m}}}\right), \quad m \geq 1 \text {. }
\end{aligned}
$$

Theorem 5 can be proved in a similar way to Theorem 4 .

Theorem 6. Suppose $z_{1}, z_{2}, \ldots, z_{s} \in \mathbf{C}(1 \leq s \leq u)$ and denote $z_{1}+$ $z_{2}+\cdots+z_{s}=z$. Let $f$ be an arithmetical function such that $f(\mathbf{m}) \neq 0$ for all $\mathbf{m} \in G$. Then for $\mathbf{n}, \mathbf{r} \in G$

$$
\begin{aligned}
& S_{A, k}^{f^{z}, \mu_{A_{k}}}\left(\mathbf{n}_{1}, \ldots, \mathbf{n}_{u} ; \mathbf{r}\right)=\sum_{\substack{\mathbf{d}_{1}, \ldots, \mathbf{d}_{s} \in A_{k}(\mathbf{r}) \\
\left[\mathbf{d}_{1}, \ldots, \mathbf{d}_{\boldsymbol{s}}\right]=\mathbf{r}}} S_{A, k}^{f^{z_{1}}, \mu_{A_{k}}}\left(\mathbf{n}_{1}, \mathbf{n}_{s+1}, \ldots, \mathbf{n}_{u} ; \mathbf{d}_{1}\right) \cdots \\
& \cdot S_{A, k}^{f^{z}, \mu_{A_{k}}}\left(\mathbf{n}_{s}, \mathbf{n}_{s+1}, \ldots, \mathbf{n}_{u} ; \mathbf{d}_{s}\right),
\end{aligned}
$$

where the symbol [‥] is used for the least common multiple.

Proof. Let $R(\mathbf{r})$ denote the right-hand side of the identity of Theorem 6 . 
Then

$$
\begin{aligned}
\sum_{\mathbf{d} \in A_{k}(\mathbf{r})} R(\mathbf{d})= & \sum_{\mathbf{d} \in A_{k}(\mathbf{r})} \sum_{\substack{\mathbf{d}_{1}, \ldots, \mathbf{d}_{s} \in A_{k}(\mathbf{d}) \\
\left[\mathbf{d}_{1}, \ldots, \mathbf{d}_{s}\right]=\mathbf{d}}} S_{A, k}^{f^{z_{1}}, \mu_{A_{k}}}\left(\mathbf{n}_{1}, \mathbf{n}_{s+1}, \ldots, \mathbf{n}_{u} ; \mathbf{d}_{1}\right) \cdots \\
& \cdot S_{A, k}^{f^{z_{s}, \mu_{A_{k}}}\left(\mathbf{n}_{s}, \mathbf{n}_{s+1}, \ldots, \mathbf{n}_{u} ; \mathbf{d}_{s}\right)} \\
= & \prod_{j=1}^{s} \sum_{\mathbf{d}_{j} \in A_{k}(\mathbf{r})} S_{A, k}^{f^{z_{j}}, \mu_{A_{k}}}\left(\mathbf{n}_{j}, \mathbf{n}_{s+1}, \ldots, \mathbf{n}_{u} ; \mathbf{d}_{j}\right) \\
= & \prod_{j=1}^{s} \chi\left(\left(\mathbf{n}_{j}, \mathbf{n}_{s+1}, \ldots, \mathbf{n}_{u}\right) ; \mathbf{r}^{k}\right) f^{z_{j}}(\mathbf{r})=\chi\left(\left(\mathbf{n}_{i}\right) ; \mathbf{r}^{k}\right) f^{z}(\mathbf{r})
\end{aligned}
$$

that is,

$$
\left(R A_{k} E\right)(\mathbf{r})=\chi\left(\left(\mathbf{n}_{i}\right) ; \mathbf{r}^{k}\right) f^{z}(\mathbf{r}),
$$

where $\chi$ is the function defined in the introduction. Thus

$$
R(\mathbf{r})=\left(\chi\left(\left(\mathbf{n}_{i}\right) ;(\cdot)^{k}\right) f^{z} A_{k} \mu_{A_{k}}\right)(\mathbf{r})=S_{A, k}^{f^{z}, \mu_{A_{k}}}\left(\mathbf{n}_{1}, \ldots, \mathbf{n}_{u} ; \mathbf{r}\right) .
$$

This completes the proof.

Remark. It is easy to see that the value of the sum

$$
S_{A, k}^{f, g}\left(\mathbf{n}_{1}, \ldots, \mathbf{n}_{u} ; \mathbf{r}\right)
$$

is independent of the order of the variables $\mathbf{n}_{1}, \ldots, \mathbf{n}_{u}$. Thus Theorems $1,4,5$ and 6 can be further generalized by rearranging the first $u$ variables into an arbitrary order.

Theorem 7. Suppose $g, h$ and $H$ are arithmetical functions such that $h$, $H$ are quasi- $A_{k}$-multiplicative and $h_{A_{k}} g H=(h g H)(\mathbf{1}) E_{0}$, where $E_{0}(\mathbf{1})=1$ and $E_{0}(\mathbf{n})=0$ for $\mathbf{n} \neq 1$. Let $f$ be an arbitrary arithmetical function. Then for $\mathbf{r} \in G, a=0,1$

$$
\sum_{\mathbf{d} \in A_{k}(\mathbf{r})} S_{A, k}^{f, g}\left(\mathbf{d}^{k^{a}} ; \mathbf{r} / \mathbf{d}\right) h(\mathbf{d}) H(\mathbf{r} / \mathbf{d})=g(\mathbf{1})(f H)(\mathbf{m}) h\left(\mathbf{m}^{k^{1-a}}\right)
$$

if $\mathbf{r}=\mathbf{m}^{k^{1-a}+1}, \mathbf{m} \in A_{k}(\mathbf{r})$, and $=0$ otherwise.

Proof. Denote by $L$ the left-hand side of (11). Then

$$
L=\sum_{\mathbf{d} \in A_{k}(\mathbf{r})} \sum_{\substack{\boldsymbol{\delta} \in A_{k}(\mathbf{r} / \mathbf{d}) \\ \boldsymbol{\delta}^{k} \mid \mathbf{d}^{k^{a}}}} f(\boldsymbol{\delta}) g(\mathbf{r} /(\mathbf{d} \boldsymbol{\delta})) h(\mathbf{d}) H(\mathbf{r} / \mathbf{d}) .
$$


It can be proved that

$$
\mathbf{d} \in A_{k}(\mathbf{r}), \quad \delta \in A_{k}(\mathbf{r} / \mathbf{d}), \quad \delta^{k} \mid \mathbf{d}^{k^{a}}
$$

if, and only if,

$$
\delta \in A_{k}(\mathbf{r}), \quad \delta^{k^{1-a}+1} \in A_{k}(\mathbf{r}), \quad \mathbf{d}=\delta^{k^{1-a}} \mathbf{e}, \quad \mathbf{e} \in A_{k}\left(\mathbf{r} / \delta^{k^{1-a}+1}\right) .
$$

Therefore

$$
\begin{aligned}
L & =\sum_{\substack{\boldsymbol{\delta} \in A_{k}(\mathbf{r}) \\
\boldsymbol{\delta}^{1-a}+1 \in A_{k}(\mathbf{r})}} \sum_{\mathbf{e} \in A_{k}\left(\mathbf{r} / \boldsymbol{\delta}^{k^{1-a}+1}\right)} f(\boldsymbol{\delta}) g\left(\left(\mathbf{r} / \boldsymbol{\delta}^{k^{1-a}+1}\right) / \mathbf{e}\right) h\left(\boldsymbol{\delta}^{k^{1-a}} \mathbf{e}\right) H\left(\mathbf{r} /\left(\boldsymbol{\delta}^{k^{1-a}} \mathbf{e}\right)\right) \\
& =\sum_{\substack{\delta \in A_{k}(\mathbf{r}) \\
\boldsymbol{\delta}^{\boldsymbol{k}^{1-a}+1} \in A_{k}(\mathbf{r})}} \frac{(f H)(\boldsymbol{\delta})}{H(\mathbf{1})} \frac{h\left(\boldsymbol{\delta}^{k^{1-a}}\right)}{h(\mathbf{1})}\left(h A_{k} g H\right)\left(\mathbf{r} / \delta^{k^{1-a}+1}\right) \\
& =\sum_{\substack{\boldsymbol{\delta} \in A_{k}(\mathbf{r}) \\
\boldsymbol{\delta}^{k^{1-a}+1} \in A_{k}(\mathbf{r})}}(f H)(\boldsymbol{\delta}) h\left(\boldsymbol{\delta}^{k^{1-a}}\right) g(\mathbf{1}) E_{0}\left(\mathbf{r} / \delta^{k^{1-a}+1}\right) .
\end{aligned}
$$

We thus arrive at our result.

Theorem 8. Suppose $f$ is a quasi- $A_{k}$-multiplicative function and $\mathbf{a}, \mathbf{b}$, $\mathbf{r} \in G$ with $\mathbf{a}, \mathbf{b} \in A_{k}(\mathbf{r})$. Then

$$
\sum_{\mathbf{d} \in A_{k}(\mathbf{r})} S_{A, k}^{f, \mu_{A_{k}}}\left((\mathbf{r} / \mathbf{d})^{k} ; \mathbf{a}\right) S_{A, k}^{f, \mu_{A_{k}}}\left((\mathbf{r} / \mathbf{b})^{k} ; \mathbf{d}\right)= \begin{cases}f(\mathbf{1}) f(\mathbf{r}) & \text { if } \mathbf{a}=\mathbf{b}, \\ 0 & \text { if } \mathbf{a} \neq \mathbf{b} .\end{cases}
$$

Theorem 9. Suppose $f$ is a quasi- $A_{k}$-multiplicative function such that $f(\mathbf{r}) \neq 0$ for all $\mathbf{r} \in G$. Then for all $\mathbf{n}, \mathbf{r} \in G$ and integers $a, b$

$$
\sum_{\mathbf{d} \in A_{k}(\mathbf{r})} S_{A, k}^{f^{a}, \mu_{A_{k}}}\left(\mathbf{d}^{k} ; \mathbf{r}\right) S_{A, k}^{f^{b}, \mu_{A_{k}}}(\mathbf{n} ; \mathbf{r} / \mathbf{d})=f^{a}(\mathbf{r}) f(\mathbf{1})^{b}\left(f^{a-b} A_{k} \mu_{A_{k}}\right)(\boldsymbol{\delta}) f^{b-a}(\boldsymbol{\delta})
$$

where $\delta^{k}=\left(\mathbf{n}, \mathbf{r}^{k}\right)_{A, k}$.

Theorem 10. Suppose $f$ is an $A_{k}$-multiplicative function with $\left(f A_{k} \mu_{A_{k}}\right)$ $(\mathbf{r}) \neq 0$ for all $\mathbf{r} \in G$. Then for all $\mathbf{m}, \mathbf{n}, \mathbf{r} \in G$

$$
\sum_{\mathbf{d} \in A_{k}(\mathbf{r})} \frac{S_{A, k}^{f, \mu_{A_{k}}}(\mathbf{n} ; \mathbf{d}) S_{A, k}^{f, \mu_{A_{k}}}(\mathbf{m} ; \mathbf{d})}{\left(f A_{k} \mu_{A_{k}}\right)(\mathbf{d})}=\frac{f(\mathbf{r})}{\left(f A_{k} \mu_{A_{k}}\right)(\mathbf{r} / \delta)}
$$

if $\left(\mathbf{n}, \mathbf{r}^{k}\right)_{A, k}=\left(\mathbf{m}, \mathbf{r}^{k}\right)_{A, k}=\delta^{k}$, and $=0$ otherwise. 
Theorem 11. Suppose $f$ is a quasi- $A_{k}$-multiplicative function and $\mathbf{n}, \mathbf{r} \in$ $G$. Denote $\mathbf{a}=\mathbf{r} / \gamma_{A_{k}}(\mathbf{r}), \mathbf{b}^{k}=\left(\mathbf{n}, \gamma(\mathbf{r})^{k}\right)_{A, k}$, where $\gamma_{A_{k}}(\mathbf{1})=\mathbf{1}$ and for $\mathbf{r} \neq \mathbf{1}$, $\gamma_{A_{k}}(\mathbf{r})$ is the product of distinct prime divisors of $\mathbf{r}$. Then

$$
f(\mathbf{1}) S_{A, k}^{f, \mu_{A_{k}}}\left(\mathbf{a}^{k} \mathbf{n} ; \mathbf{r}\right)=f(\mathbf{a}) \mu_{A_{k}}\left(\gamma_{A_{k}}(\mathbf{r})\right) \mu_{A_{k}}(\mathbf{b})\left(f{A_{k}}_{\mu_{A_{k}}}\right)(\mathbf{b}) .
$$

By quasi-multiplicativity Theorems $8-11$ can be proved by considering the case in which $\mathbf{r}$ is a prime power. We omit the details.

Theorem 12. Suppose $f, g, h$ and $H$ are arithmetical functions and $\mathbf{n} \in G$. Let $w$ denote the arithmetical function such that $w(\mathbf{1})=0$ and for $\mathbf{r} \neq \mathbf{1}, w(\mathbf{r})$ is the number of distinct prime divisors of $\mathbf{r}$. Then

$$
\sum_{\mathbf{d}_{1}, \ldots, \mathbf{d}_{u}, \mathbf{e}} S_{A, k}^{f, g}\left(\mathbf{d}_{1}, \ldots, \mathbf{d}_{u} ; \mathbf{e}\right) h(\mathbf{e}) H(\mathbf{n} / \mathbf{e})=f(\mathbf{1})\left(\left(u^{w} H\right) U(g h)\right)(\mathbf{n}),
$$

where the summation is over $\mathbf{d}_{1}, \ldots, \mathbf{d}_{u}, \mathbf{e} \in G$ such that $\mathbf{d}_{1} \cdots \mathbf{d}_{u} \mathbf{e}=\mathbf{n}$ and $\mathbf{d}_{1}, \ldots, \mathbf{d}_{u}, \mathbf{e}$ are pairwise relatively prime.

Proof. The left-hand side of the identity in Theorem 12 is

$$
\begin{aligned}
\sum_{\mathbf{d}_{1}, \ldots, \mathbf{d}_{u}, \mathbf{e}} f(\mathbf{1}) g(\mathbf{e}) h(\mathbf{e}) H(\mathbf{n} / \mathbf{e}) & =f(\mathbf{1}) \sum_{\mathbf{e} \in U(\mathbf{n})} u^{w(\mathbf{n} / \mathbf{e})} g(\mathbf{e}) h(\mathbf{e}) H(\mathbf{n} / \mathbf{e}) \\
& =f(\mathbf{1})\left((g h) U\left(u^{w} H\right)\right)(\mathbf{n})
\end{aligned}
$$

hence the theorem is valid. Then

Theorem 13. Let $f, g, h$ and $H$ be arithmetical functions and $\mathbf{n} \in G$.

$$
\sum_{\substack{\mathbf{d}_{1} \cdots \mathbf{d}_{u} \mathbf{e}=\mathbf{n} \\\left(\mathbf{d}_{1} \cdots \mathbf{d}_{u}, \mathbf{e}\right)=\mathbf{1}}} S_{A, k}^{f, g}\left(\mathbf{d}_{1}, \ldots, \mathbf{d}_{u} ; \mathbf{e}\right) h(\mathbf{e}) H(\mathbf{n} / \mathbf{e})=f(\mathbf{1})\left(\left(E_{u} H\right) U(g h)\right)(\mathbf{n}),
$$

where $E_{u}=E_{D} E_{D} \cdots D E$ ( $u$ factors).

Proof. The left-hand side of the identity is

$$
\begin{array}{r}
\sum_{\substack{\mathbf{d}_{1} \cdots \mathbf{d}_{u} \mathbf{e}=\mathbf{n} \\
\left(\mathbf{d}_{1} \cdots \mathbf{d}_{u}, \mathbf{e}\right)=\mathbf{1}}} f(\mathbf{1}) g(\mathbf{e}) h(\mathbf{e}) H(\mathbf{n} / \mathbf{e})=f(\mathbf{1}) \sum_{\mathbf{e} \in U(\mathbf{n})}\left(\sum_{\mathbf{d}_{1} \cdots \mathbf{d}_{u}=\mathbf{n} / \mathbf{e}} 1\right) g(\mathbf{e}) h(\mathbf{e}) H(\mathbf{n} / \mathbf{e}) \\
=f(\mathbf{1}) \sum_{\mathbf{e} \in U(\mathbf{n})} E_{u}(\mathbf{n} / \mathbf{e}) g(\mathbf{e}) h(\mathbf{e}) H(\mathbf{n} / \mathbf{e})=f(\mathbf{1})\left(\left(E_{u} H\right) U(g h)\right)(\mathbf{n}) .
\end{array}
$$

We thus arrive at our result. 
Remark. A large number of special cases of our results can be found in the literature. In fact, special cases of Theorem 1 can be found in [33, Corollary (2.1.5), p. 170, Theorem (2.2.6), p. 174], [38, pp. 15 and 72] and [40, Chapter 4.1]. Special cases of Theorem 2 can be found in [2, equation (2.7)], [20, equation (6)], [33, Theorem (2.2.12), p. 176], [34, Theorem 3.1], [35, equation (4.3)], [41, Theorem 3] and [44, Theorem 5.3]. Special cases of Theorem 3 can be found in $[1$, Theorems 1-4], [2, equations (2.8), (2.10)], [4, Theorem 8], [5, Corollaries $2,3,4,5,10.2$ and 10.4$]$, [6, equation (5.4)], [8, equations $(3.1),(5.1)],[10, \mathrm{p}$. 203], [12, equation (3.16)], [18, equation (2.3), p. 194], [20, equations (4), (5)], [23, Lemma 1], [28, Theorem 1], [29, equation (1a) and Theorem 8], [30, equation (2.6)], [31, Theorem 2.4], [33, Theorem (2.1.8), p. 172, Theorem (2.2.11), p. 176], [34, Theorems 3.2, 3.3], [36, equation (3.3)], [41, Theorems 1, 2] and [44, Theorem 5.4]. Special cases of Theorem 4 can be found in [1, Theorems 5, 6], [28, Theorem 2] and [29, Theorem (1b)]. Special cases of Theorem 5 can be found in $[1$, Theorems $7,8]$, [2, Theorem 3.2], [3, Corollary 2.1], [17, Lemma 2.6], [29, Theorem 3], [40, Theorem 4.1.2] and [44, Theorem 5.5]. Special cases of Theorem 6 can be found in [7, Lemma 4], [14, Lemma 3] and [25, Theorem 8]. Special cases of Theorem 7 can be found in [2, Theorem 2.6], [16, Theorem 1], [20, equation (3)], [37, equations $(2.10),(2.11)]$ and $[40$, equation (4.14)]. Special cases of Theorem 8 can be found in [3, Theorem 2], [8, equation (4.2)], [15, Theorem 1], [18, Lemma 2.2, p. 194], [21, Theorem 5], [24, Theorem 3], [26, equation (4.1.6)] and [32, Theorem 7.2]. A special case of Theorem 9 can be found in [37, equation (2.12)]. Special cases of Theorem 10 can be found in [4, Theorem 6], [8, equation (4.5)], [9, Theorem 3.3], [21, Theorem 4], [32, Theorem 7.4] and [34, Theorem 3.4]. Special cases of Theorem 11 can be found in [11, Theorem 3] and [25, Theorem 3]. Finally, Theorem 6 of [25] is a special case of Theorems 12 and 13.

\section{Totally $A-k$-even functions $(\bmod r)$}

Let $\mathbf{r} \in G$ be fixed. Then an arithmetical function $f(\mathbf{n} ; \mathbf{r})$ of one variable is said to be $A$-k-even $(\bmod \mathbf{r})$ if $f(\mathbf{n} ; \mathbf{r})=f\left(\left(\mathbf{n}, \mathbf{r}^{k}\right)_{A, k} ; \mathbf{r}\right)$ for all $\mathbf{n} \in G$. An arithmetical function $f\left(\mathbf{n}_{1}, \ldots, \mathbf{n}_{u} ; \mathbf{r}\right)$ of $u$ variables is said to be totally $A$ $k$-even $(\bmod \mathbf{r})$ if there exists an $A$-k-even function $g(\mathbf{n} ; \mathbf{r})(\bmod \mathbf{r})$ such that $f\left(\mathbf{n}_{1}, \ldots, \mathbf{n}_{u} ; \mathbf{r}\right)=g\left(\left(\mathbf{n}_{1}, \ldots, \mathbf{n}_{u}\right) ; \mathbf{r}\right)$ for all $\mathbf{n}_{1}, \ldots, \mathbf{n}_{u} \in G$. The concept of a totally $A$ - $k$-even function $(\bmod \mathbf{r})$ originates from $[14]$ in the case of the arithmetical semigroup of positive integers. It can be proved (cf. [14, Theorem 1]) that an arithmetical function $f\left(\mathbf{n}_{1}, \ldots, \mathbf{n}_{u} ; \mathbf{r}\right)$ is totally $A$ - $k$-even $(\bmod \mathbf{r})$ if, and only if, it has a unique representation of the form

$$
f\left(\mathbf{n}_{1}, \ldots, \mathbf{n}_{u} ; \mathbf{r}\right)=\sum_{\mathbf{d} \in A_{k}(\mathbf{r})} \alpha(\mathbf{d} ; \mathbf{r}) C_{A, k}\left(\mathbf{n}_{1}, \ldots, \mathbf{n}_{u} ; \mathbf{d}\right),
$$

where 


$$
\alpha(\mathbf{d} ; \mathbf{r})=\mathbf{r}^{-k u} \sum_{\delta \in A_{k}(\mathbf{r})} g\left(\mathbf{r}^{k} / \boldsymbol{\delta}^{k} ; \mathbf{r}\right) C_{A, k}\left(\mathbf{r}^{k} / \mathbf{d}^{k}, \ldots, \mathbf{r}^{k} / \mathbf{d}^{k} ; \boldsymbol{\delta}\right) .
$$

The coefficients $\alpha(\mathbf{d} ; \mathbf{r})$ are called the Fourier coefficients of $f\left(\mathbf{n}_{1}, \ldots, \mathbf{n}_{u} ; \mathbf{r}\right)$. It can also be proved (cf. [14, Theorem 2]) that an arithmetical function $f\left(\mathbf{n}_{1}, \ldots, \mathbf{n}_{u} ; \mathbf{r}\right)$ is totally $A$ - $k$-even $(\bmod \mathbf{r})$ if, and only if, it has the form

$$
f\left(\mathbf{n}_{1}, \ldots, \mathbf{n}_{u} ; \mathbf{r}\right)=\sum_{\mathbf{d}^{k} \in A\left(\left(\left(\mathbf{n}_{i}\right), \mathbf{r}^{k}\right)_{A, k}\right)} f^{\prime}(\mathbf{d} ; \mathbf{r})
$$

In this case

$$
\alpha(\mathbf{d} ; \mathbf{r})=\mathbf{r}^{-k u} \sum_{\mathbf{e} \in A_{k}(\mathbf{r} / \mathrm{d})} f^{\prime}(\mathbf{r} / \mathbf{e} ; \mathbf{r}) \mathbf{e}^{k u}
$$

By (12) we find that the generalized Ramanujan's sum considered in this paper is a totally $A$ - $k$-even function $(\bmod \mathbf{r})$.

The purpose of this section is to note that the equations (8) and (10) can be extended to totally $A$ - $k$-even functions $(\bmod \mathbf{r})$. In fact, if we replace the generalized Ramanujan's sum by an arbitrary totally $A$ - $k$-even function $f\left(\mathbf{n}_{1}, \ldots, \mathbf{n}_{u} ; \mathbf{r}\right)$ $(\bmod \mathbf{r})$ in the left-hand sides of equations $(8)$ and $(10)$, we must replace the factor of $f(\mathbf{d}) g(\mathbf{r} / \mathbf{d})$ by $f^{\prime}(\mathbf{d} ; \mathbf{r})$ in the right-hand sides of the equations.

\section{References}

[1] Apostol, T.M.: Arithmetical properties of generalized Ramanujan sums. - Pacific J. Math. 41, 1972, 281-293.

[2] Chidambaraswamy, J.: Generalized Ramanujan's sum. - Period. Math. Hungar. 10, 1979, 71-87.

[3] Cohen, E.: An extension of Ramanujan's sum. II. Additive properties. - Duke Math. J. $22,1955,543-550$.

[4] Cohen, E.: An extension of Ramanujan's sum. III. Connections with totient functions. Duke Math. J. 23, 1956, 623-630.

[5] Cohen, E.: Representations of even functions $(\bmod r)$, I. Arithmetical identities. - Duke Math. J. 25, 1958, 401-421.

[6] Cohen, E.: A class of residue systems $(\bmod r)$ and related arithmetical functions, I. A generalization of Möbius inversion. - Pacific J. Math. 9, 1959, 13-23.

[7] Cohen, E.: A class of arithmetical functions in several variables with applications to congruences. - Trans. Amer. Math. Soc. 96, 1960, 355-381.

[8] Cohen, E.: On the inversion of even functions of finite abelian groups $(\bmod H)$. - J. Reine Angew. Math. 207, 1961, 192-202.

[9] Cohen, E.: Unitary functions $(\bmod r)$. - Duke Math. J. 28, 1961, 475-485.

[10] Delsarte, J.: Essai sur l'application de la theorie des fonctions presque périodiques a l'arithmétique. - Ann. Sci. École Norm. Sup. 62, 1945, 185-204.

[11] Donovan, G.S., and D. Rearick: On Ramanujan's sum. - Norske Vid. Selsk. Forh. 39, 1966, 1-2. 
[12] Hanumanthachari, J., and V.V. Subrahmanya Sastri: Certain totient functionsallied Ramanujan sums and applications to certain linear congruences. - Math. Student 42, 1974, 214-222.

[13] Haukkanen, P.: Classical arithmetical identities involving a generalization of Ramanujan's sum.- Ann. Acad. Sci. Fenn. Ser. A. I. Math. Dissertationes 68, 1988, 1-69.

[14] Haukkanen, P. and P.J. McCarthy: Sums of values of even functions . - Portugal. Math. (To appear.)

[15] Horadam, E.M.: Ramanujan's sum for generalised integers. - Duke Math. J. 31, 1964, 697-702.

[16] Horadam, E.M.: Exponential functions for arithmetical semi-groups. - J. Reine Angew. Math. 222, 1966, 14-19.

[17] Johnson, K.R.: Reciprocity and multiplicativity in a class of arithmetic functions. - $\mathrm{Ph}$. D. thesis, University of Colorado, 1980.

[18] Knopfmacher, J.: Abstract analytic number theory. - North-Holland, Amsterdam-New York, 1975.

[19] LahiRI, D.B.: Hypo-multiplicative number-theoretic functions. - Aequationes Math. 9, 1973, 184-192.

[20] MCCarthy, P.J.: Some properties of the extended Ramanujan sums. - Arch. Math. 11, 1960, 253-258.

[21] MCCARThy, P.J.: Regular arithmetical convolutions. - Portugal. Math. 27, 1968, 1-13.

[22] MCCARThy, P.J.: Introduction to arithmetical functions. - Springer-Verlag, New YorkBerlin-Heidelberg-Tokyo, 1986.

[23] NAGEswara RAO, K.: Unitary class division of integers $\bmod n$ and related arithmetical identities. - J. Indian Math. Soc. 30, 1966, 195-205.

[24] Nageswara Rao, K.: Some applications of Carlitz's $\eta$-sum. - Acta Arith. 12, 1967, 213-221.

[25] Nageswara Rao, K.: Some identities involving an extension of Ramanujan's sum. Norske Vid. Selsk. Forh. 40, 1967, 18-23.

[26] Nageswara RaO, K., and R. Sivaramakrishnan: Ramanujan's sum and its applications to some combinatorial problems. - Congr. Numer. 31, 1981, 205-239.

[27] Narkiewicz, W.: On a class of arithmetical convolutions. - Colloq. Math. 10, 1963, 81-94.

[28] Nicol, C.A.: On restricted partitions and a generalization of the Euler $\Phi$ number and the Moebius functions. - Proc. Nat. Acad. Sci. U.S.A. 39, 1953, 963-968.

[29] Nicol, C.A., and H.S. Vandiver: A von Sterneck arithmetical function and restricted partitions with respect to a modulus. - Proc. Nat. Acad. Sci. U.S.A. 40, 1954, 825835 .

[30] Ramanujan, S.: On certain trigonometrical sums and their applications in the theory of numbers. - Trans. Cambridge Philos. Soc. 22, 1918, 259-276.

[31] Shader, L.E.: Arithmetic functions associated with unitary divisors in $G F[q, x]$, I. - Ann. Mat. Pura Appl. 86, 1970, 79-85.

[32] Sita Ramaiah, V.: Arithmetical sums in regular convolutions. - J. Reine Angew. Math. 303/304, 1978, 265-283.

[33] Sivaramakrishnan, R.: Contributions to the study of multiplicative arithmetic functions. - Ph. D. thesis, University of Kerala, 1971.

[34] Sivaramakrishnan, R.: Multiplicative even functions $(\bmod r)$. II. Identities involving Ramanujan sums. - J. Reine Angew. Math. 302, 1978, 44-50. 
[35] Sivaramakrishnan, R.: On abstract Möbius inversion. - Proceedings of the 2nd Conference on Number Theory (Ootacamund, 1980), Matscience Rep. 104, 85-100.

[36] Subrahmya Sastri, V. V.: On a certain totient function for generalised integers. - Math. Student 37, 1969, 59-66.

[37] Sugunamma, M.: Eckford Cohen's generalizations of Ramanujan's trigonometrical sum $C(n, r)$. - Duke Math. J. 27, 1960, 323-330.

[38] Sugunamma, M.: Contribution to the study of general arithmetic functions. - Ph. D. thesis, Sri Venkateswara University, Tirupati, 1965.

[39] Vaidyanathaswamy, R.: The theory of multiplicative arithmetic functions. - Trans. Amer. Math. Soc. 33, 1931, 579-662.

[40] Venkataraman, C.S.: A new identical equation for multiplicative functions of two arguments and its applications to Ramanujan's sum $C_{M}(N)$. - Proc. Indian Acad. Sci. Sect. A 24, 1946, 518-529.

[41] Venkataraman, C.S.: Further applications of the identical equation of Ramanujan's sum $C_{M}(N)$ and Kronecker's function $\varrho(M, N)$. - J. Indian Math. Soc. 10, 1946, 57-61.

[42] Venkataraman, C.S.: The ordinal correspondence and certain classes of multiplicative functions of two variables. - J. Indian Math. Soc. 10, 1946, 81-101.

[43] Venkataraman, C.S.: On von Sterneck-Ramanujan function. - J. Indian Math. Soc. 13, 1949, 65-72.

[44] Venkataraman, C.S., and R. Sivaramakrishnan: An extension of Ramanujan's sum. - Math. Student 40 A, 1972, 211-216.

[45] Yocom, K.L.: Totally multiplicative functions in regular convolution rings. - Canad. Math. Bull. 16, 1973, 119-128.

University of Tampere

Department of Mathematical Sciences

P. O. Box 607

SF-33101 Tampere

Finland

Received 9 December 1988 